\title{
A saúde dos bombeiros militares no combate a COVID-19 no Brasil
}

\author{
The health of military firefighters in the fight against COVID-19 in Brazil
}

\author{
Vinicius Sonoda de Freitas ${ }^{\circ}$ \\ Centro Universitário de Maringá, Departamento de Educação Física. Maringá, Paraná, Brasil. *Autor para correspondência: E-mail: \\ vinsf20@hotmail.com
}

\begin{abstract}
Resumo: Introdução: O Bombeiro Militar, no exercício da sua atividade, coloca sua vida em risco para salvar a vida de terceiros ou defender bens públicos e privados da sociedade. E tem sido assim desde o surgimento da Covid-19 no Brasil, com as medidas restritivas, isolamentos ou ações para se evitar a contaminação desses profissionais e da população. E é inevitável que alguns bombeiros sejam infectados, afinal, tanto os combatentes quanto aqueles que dão suporte à linha de frente, estão em situação de risco. Objetivo do estudo é descrever a importância dos bombeiros militares no combate à pandemia, abordando as taxas de contaminação e medidas preventivas adotadas por alguns Estados brasileiros. Revisão: $\mathrm{O}$ tema é de grande relevância, uma vez que a Covid-19 é um vírus com grande potência e que vem causando um dos mais sérios problemas de saúde pública e econômica em escala global. A metodologia baseou-se em uma revisão de literatura, utilizando banco de dados da Scielo, PubMed e Biblioteca Virtual em Saúde (BVS) e Science Direct, publicados até setembro de 2020 no Brasil. Discussão: Devido à alta incidência e mortalidade, os efeitos da Covid-19 deverão ser sentidos por muito tempo e medidas de suporte de apoio emocional com psicólogos e outras formas de assistência médica e social aos bombeiros militares são fundamentais e devem ser realizadas em todos os Estados brasileiros. Considerações finais: De acordo com as diversas fontes utilizadas neste estudo, pode-se verificar que conviver com a Covid-19 é adaptar-se a um novo estilo de vida para prevenir a reincidência da doença até o surgimento da vacina para a população.
\end{abstract}

Palavras-chave: bombeiros, Covid-19, saúde pública.

\begin{abstract}
Introduction: The Firefighter Military, in the exercise of the activity, puts his life at risk to save the lives of others or to defend public and private assets of society. And this has been the case since the emergence of Covid-19 in Brazil, with restrictive measures, isolations or actions to avoid contamination of these professionals and the population. And it is inevitable that some firefighters are infected, after all, both combatants and those who support the front line are at risk. Objective of the study is to describe the importance of military firefighters in combating the pandemic, addressing contamination rates and preventive measures adopted by some Brazilian states. Review: The theme it is of great relevance, since Covid-19 is a highly potent virus that has been causing one of the most serious public and economic health problems on a global scale. The methodology was based on a literature review, using databases from Scielo, PubMed and the Virtual Health Library (VHL) and Science Direct, published until September 2020 in Brazil. Discussion: Due to the high incidence and mortality, the effects of Covid-19 can be felt for a long time and emotional support measures with psychologists and other forms of medical and social assistance to military firefighters are essential and must be carried out in all Brazilian states. Final Considerations: According to the various sources used in this study, it can be seen that living with Covid19 is adapting to a new lifestyle to prevent the recurrence of the disease until the vaccine appears for the population.
\end{abstract}

Keywords: firefighters, Covid-19, public health.

\section{Introdução}

SARS-CoV-2/2019-nCoV é a sigla utilizada para distinguir um novo coronavírus capaz de infectar humanos, podendo levar ao desenvolvimento da síndrome respiratória aguda grave (SRAG) e que vem causando um dos mais sérios problemas de saúde pública e econômico em escala global dos últimos 100 anos (Liu et al., 2020). A doença causada pelo vírus, conhecida como Covid-19, teve seu primeiro surto em dezembro de 2019 na cidade de Wuhan, província de Hubei na China. Apesar dos esforços das autoridades chinesas para conter o surto, houve uma rápida disseminação do vírus em escala global, levando a 
Organização Mundial da Saúde (OMS) a declarar em 30 de janeiro de 2020 a Emergência de Saúde Pública de Importância Internacional (ESPII), o mais alto nível de alerta da organização, e pandemia em 11 de março de 2020 (WHO, 2020; Shereen et al., 2020).

O SARS-CoV-2 tem alto poder de transmissão, sendo que cada pessoa infectada, em média, pode transmitir o vírus para outras três (Cespedes \& Pires, 2020). A transmissão pode ocorrer de forma direta, por meio do contato com gotículas respiratórias de uma pessoa infectada, produzidas ao falar, tossir ou espirrar, ou de forma indireta quando superfícies contaminadas são tocadas e as mãos são levadas aos olhos, boca ou nariz. Há evidências de que o vírus possa ficar suspenso no ar na forma de aerossóis por até três horas, caracterizando sua possível transmissão aérea (Correia et al., 2020). Os sintomas mais comuns da doença são febre, cansaço e tosse seca, podendo haver perda de olfato e paladar. Mais de $80 \%$ da população acometida apresenta sintomas que variam de leves a moderados, ainda podendo ser imperceptíveis quando assintomáticos. Contudo, entre 15 e $20 \%$ dos doentes está susceptível a necessidade de atendimento hospitalar, sendo que uma fração menor desenvolve SRAG com alto risco de evoluir para óbito (Freitas, 2020; Moghadas et al., 2020). Devido à alta capacidade de transmissão do vírus e o colapso iminente na capacidade de atendimento hospitalar, a OMS orientou que todos os países afetados adotassem o distanciamento social como única estratégia eficaz a ser empregada com a finalidade de retardar o contágio a tempo dos sistemas de saúde socorrerem a população adequadamente. Outras medidas complementares foram adotadas seguindo as orientações da OMS, como a higienização frequente das mãos e de superfícies frequentemente tocadas, assim como de embalagens alimentícias, frutas, verduras e legumes, além do amplo uso de máscaras faciais pela população em geral.

Apesar de todos os esforços empregados, até setembro de 2020, o Coronavirus Datacenter do John Hopkins Hospital contabilizava que o novo coronavírus já havia infectado ao redor de 30 milhões de pessoas e causado a morte de 1 milhão em 213 países, desconsiderando-se as subnotificações inerentes ao monitoramento de doenças infecciosas, especialmente quando se considera a escala global. O Brasil é um dos países mais afetados pela pandemia do novo coronavírus. Seis meses após a pandemia ser declarada pela OMS, mais de 130 mil brasileiros desenvolveram complicações graves da doença e evoluíram para óbito, colocando o país em segundo lugar em número de mortes por Covid-19 e entre os três primeiros em número de infectados, ao lado dos Estados Unidos e Índia.

Na linha de frente do combate a Covid-19 estão os profissionais da saúde. Segundo o Relatório Mundial de Saúde (2006), a OMS define os recursos humanos da saúde como: "todas as pessoas engajadas em ações cuja intenção principal é a melhoria da saúde”. Dentro dessa definição, há um amplo espectro de profissionais que desempenham as mais distintas funções, dentre eles: médicos, enfermeiros, técnicos e assistentes em enfermagem, dentistas, farmacêuticos, radiologistas, fisioterapeutas, psicólogos, nutricionistas, estudantes da área de saúde e profissionais administrativos do setor de saúde. Segundo reconhecimento da própria OMS, muitas outras ocupações laborais, remuneradas ou não, são inclusivas nesta definição, embora nem sempre diretamente ligadas à saúde. Estas incluem cuidadores de idosos e doentes, parteiras e doulas, gestores de unidades de saúde, assistentes sociais, operadores de equipamentos médicos, técnicos de laboratórios, socorristas de ambulâncias, entre outros. Dentre esses profissionais incluem-se os militares do Corpo de Bombeiros, tanto os que desempenham serviços operacionais de socorro como os que trabalham na retaguarda dos serviços administrativos.

De um modo geral, todos os profissionais da saúde são mais susceptíveis ao risco de contaminação cruzada a partir da população atendida, uma vez que estão diretamente em contato com os doentes e expostos a locais e objetos contaminados por longos períodos de tempo. Aos fatores de risco ocupacionais, além da maior exposição à contaminação, somam-se ainda a disponibilidade limitada de equipamentos de proteção individual (EPI) em relação à demanda profissional, a sobrecarga de trabalho, a falta de capacitação suficiente quanto ao uso adequado de medidas de proteção e de rigor nos hábitos de biossegurança (Sant'ana et al., 2020). Essas condições colocam os profissionais da linha de frente em situação de estresse extremo, potencialmente afetando sua saúde física e mental (Greenberg et al., 2020). Dentre as consequências de médio a longo-prazo da pandemia, estima-se que haja um aumento de doenças mentais incapacitantes e da taxa de suicídios entre profissionais da saúde (Troyer et al., 2020).

A pandemia de Covid-19 impulsionou a rápida produção e divulgação de conhecimento científico no mundo todo, sobre os mais diversificados aspectos da doença. Até a primeira quinzena de setembro, 21.485 estudos sobre Covid-19 retornaram na base de dados científicos Science Direct, o que equivale à 
impressionante média de 84,6 publicações/dia no ano de 2020. Dentre esses estudos, muitos têm procurado esclarecer os riscos à saúde e segurança dos profissionais da saúde, especificamente da área médica e da enfermagem, porém com menor dimensionamento dos riscos voltados para os demais trabalhadores da linha de frente da Covid-19. Por exemplo, os riscos de infecção do vírus entre bombeiros e policiais que interagem diretamente com a população não têm sido calculados, assim como para outros diversos profissionais no enfrentamento a Covid-19. Essa é uma falha significativa a favor da cadeia de transmissão do vírus, pois tanto a população infectada pode transmitir o vírus para esses profissionais, como os próprios profissionais podem atuar como disseminadores do vírus para a população susceptível. Ademais, a baixa no corpo efetivo de trabalhadores em função de afastamentos por suspeita ou confirmação da infecção pode resultar em sérios prejuízos no atendimento da população.

O trabalho tem, portanto, como objetivo compreender como os fatores relacionados, anteriormente, influenciam na qualidade de vida dos bombeiros militares. Além disso, pretende-se discutir como o enfrentamento da doença interfere na qualidade de vida desses profissionais, e mostrar a importância do apoio, seja por meio de treinamentos quantos ao uso de equipamentos de proteção individual, seja no monitoramento das taxas de infecções ao longo do tempo.

\section{Revisão}

O tema é de grande relevância, uma vez que a Covid-19 é um vírus com grande potência e que vem causando um dos mais sérios problemas de saúde pública e econômica em escala global. $\mathrm{O}$ efeito dos sintomas, a confirmação da doença e o tratamento impactam diretamente na qualidade de vida dos indivíduos.

A metodologia baseou-se em uma revisão de literatura, utilizando sites específicos, como o banco de dados da Scielo, PubMed e Biblioteca Virtual em Saúde (BVS) e Science Direct, publicados até setembro de 2020 no Brasil. Considerando os filtros utilizados, foram encontrados até a primeira quinzena de setembro, 21.485 estudos sobre a Covid-19, dos quais foram selecionadas 31 publicações, por serem mais adequadas ao tema proposto.

O presente estudo compilou informações sobre o enfrentamento por parte de militares do Corpo de Bombeiros no combate a Covid-19. De acordo com esse material disponível, é possível observar que muitas ações direcionadas ao suporte e capacitação de bombeiros para o atendimento de pacientes de Covid-19 têm sido realizadas em diversos municípios e Estados brasileiros.

No Distrito Federal, 36 militares do Corpo de Bombeiros receberam treinamento técnico ministrado por médicos e enfermeiros, no qual foram abordados temas como o uso de ventilação mecânica, cardioversão, desfibrilação, intubação de pacientes, transporte de crianças e uso correto de EPI. A ação foi decorrente de uma parceria entre o Samu e a corporação de bombeiros visando aumentar a frota de veículos disponíveis para atendimento móvel durante a pandemia (Ferraz, 2020). A parceria com o Samu também tem ocorrido em outros Estados, como a Paraíba, por meio de palestras educativas no sentido de capacitar o corpo efetivo pertencente a todos os comandos regionais dos bombeiros quanto ao uso correto de EPI e os procedimentos de desinfecção de viaturas (CBM-PB, 2020). No Tocantins, o Corpo de Bombeiros vem atuando nas ações de sanitização de 27 cidades do interior do Estado (Machado, 2020) e em Minas Gerais, as ambulâncias de resgate do Corpo de Bombeiros foram autorizadas pelo governo estadual a realizar o transporte de pacientes com Covid-19 entre municípios que não dispõem de leitos de Unidade de Terapia Intensiva (Oliveira, 2020). Em Campo Grande, o quartel do Corpo de Bombeiros foi adaptado para a realização da testagem da população em um sistema Drive-Thru, ampliando em mais de mil testes por semana a capacidade total do município (Frias, 2020).

Uma estratégia preventiva que tem sido adotada por diversos municípios tem sido a testagem em massa, tanto dos bombeiros, como dos policiais civis e militares. No município de Poços de Caldas, em Minas Gerais, os testes sorológicos realizados pela Secretaria Municipal de Saúde têm auxiliado a corporação dos bombeiros a avaliar a eficácia das medidas de desinfecção de viaturas e equipamentos utilizados durante o atendimento à população (Poços Já, 2020). Em Florianópolis, tem ocorrido o monitoramento periódico desses profissionais através de testes sorológicos doados por entidades sociais e empresas em parceria com a prefeitura (ND mais, 2020).

Segundo dados da Polícia Militar e da Associação dos Praças de Santa Catarina, até o mês de julho, 498 policiais e 130 bombeiros foram afastados de suas atividades em todo o Estado (Malinverni, 2020). De 
acordo com o autor, número de profissionais infectados dobrou em apenas 15 dias, demonstrando a importância no monitoramento periódico e a vulnerabilidade desse grupo de profissionais à infecção por Covid-19. Em Blumenau, o avanço da doença resultou no fechamento temporário de um dos pelotões do Corpo de Bombeiros como medida preventiva para garantia de reserva operacional para cobrir eventuais afastamentos decorrentes de suspeita ou confirmação da infecção por Covid-19 (Elias, 2020). No Paraná, até o mês de setembro, 78 bombeiros contaminados, 75 bombeiros recuperados, 3 bombeiros foram afastados de suas atividades e nenhum óbito (CCB-BM1, 2020). Em Boa Vista, Roraima, a testagem em massa no início de setembro revelou que 87 profissionais, de um total de 293 bombeiros militares testados, já haviam contraído o vírus, o que representou cerca de 30\% de seu efetivo operacional (Gondim, 2020). No Estado do Piauí, o percentual de infectados no Corpo de Bombeiros chegou a 9\% no início de junho (Pimentel, 2020) e no Distrito Federal, ainda em abril, até $12 \%$ dos casos de Covid-19 foram em servidores da segurança pública (G1, 2020). Ainda no primeiro trimestre da pandemia, o Corpo de Bombeiros do Rio de Janeiro e do Distrito Federal, haviam afastado 628 bombeiros e 326 profissionais da segurança pública, respectivamente (Andrade, 2020).

São inúmeras as notícias sobre a contaminação de servidores do Corpo de Bombeiros e demais policiais civis e militares em todos os municípios e Estados brasileiros. Contudo, não há qualquer ação integrada do Governo Federal, através do Ministério da Saúde ou Ministério do Trabalho, no sentido de medir as taxas de infecção e de mortalidade nesses profissionais e demais ocupações dos trabalhadores da saúde, bem como dar transparência dos dados oficiais a fim de que medidas protetivas mais específicas e ajustadas com a atividade laboral possam sem adotadas (Silva \& Nobre, 2020). Na ausência desses dados oficiais, as altas taxas de contaminação têm sido noticiadas pela imprensa e deixam claro que as medidas protetivas adotadas não têm sido suficientes, o que coloca em risco tanto os profissionais, como a própria população atendida. Um bom monitoramento integrado em nível nacional ou mesmo estadual poderia destacar os municípios com maior incidência de bombeiros e demais profissionais da saúde infectados e rapidamente identificar as falhas cometidas, seja na execução dos protocolos de segurança, na sobrecarga de trabalho, na infraestrutura ou qualquer outro fator que possa ser utilizado no gerenciamento de risco (Baker et al., 2020). Além disso, é previsto que situações de crises sanitárias sejam desafios cada vez mais frequentes a serem enfrentados pelas diversas nações no futuro. A criação de planos integrados de gestão de riscos para as diferentes categorias de profissionais da saúde durante a pandemia de Covid-19 poderia resultar em aprendizagens mútuas no âmbito nacional, nos preparando para ações mais rápidas e mais eficientes em situações de novas pandemias.

Um aspecto positivo a ser destacado é que o estado de calamidade pública gerado pela pandemia de Covid-19 acabou resultando na ampliação de pessoal e infraestrutura em alguns batalhões. No Estado do Amazonas, foram nomeados 820 profissionais da saúde aprovados no concurso do Corpo de Bombeiros, que aguardavam a ocupação de seus cargos desde que o concurso público foi homologado em março de 2010 , dentre eles: médicos, enfermeiros, dentistas, farmacêuticos, assistentes sociais, motoristas e socorristas. No Espírito Santo, o Corpo de Bombeiros recebeu 20 novas viaturas de resgate, representando a maior aquisição de viaturas na história da instituição em seus 107 anos de existência (Em Dia, 2020). No caso do Amazonas, após realizar um curso preparatório para médicos da carreira militar, os médicos concursados assumiram suas atividades no hospital destinado a casos graves de Covid-19, em caráter de prontidão estratégica, assim como os demais profissionais do Corpo de Bombeiros. Almeida (2020) aponta que "trabalhadores inexperientes precisam focar a atenção no objetivo e executar a tarefa de modo lento para evitar falhas” e que "treinamentos bem-sucedidos precisam ressaltar o fato de que a evolução da pandemia é mais rápida do que a das medidas de prevenção aos novos riscos” de modo que “o objetivo buscado é desenvolver competências de reconhecimento de riscos ao lidar com situações de mudanças". Em um momento de alta complexidade na saúde pública, é possível inferir que um breve treinamento, direcionado à carreira militar, possa ser capaz de garantir segurança aos novos profissionais da saúde? Seriam eles capazes de reconhecer os riscos e interferir de maneira eficaz? Qual seu preparo físico e emocional para lidar com situações de estresse extremo em uma pandemia?

Embora grande parte de suas atividades esteja atualmente relacionada com o suporte à prevenção e ao salvamento de vítimas da Covid-19, profissionais do Corpo de Bombeiros ainda precisam atender as demandas naturais de suas funções. Este é o caso do combate ao fogo, seja este natural, acidental ou criminoso. Com o avanço dos incêndios florestais que tem ocorrido no Brasil nos últimos meses, tanto na Amazônia como no Pantanal, há um risco potencialmente maior dos bombeiros florestais desenvolverem sintomas graves de Covid-19. Uma reportagem do blog americano The Conversation, de 11 de setembro de 2020, explica, com base em evidências científicas, que a fumaça de incêndios florestais produz partículas 
muito finas que, ao serem inaladas, suprimem o sistema imunológico, aumentando o risco de doenças respiratórias, podendo tornar o novo coronavírus particularmente letal para os bombeiros. Durante a pandemia de Covid-19, o combate aos incêndios florestais tem seguido protocolos diferenciados por meio do uso de equipamentos de proteção individual e da formação de acampamentos em pequenos grupos isolados de bombeiros que desempenham suas atividades em conjunto, estes equipados com banheiros e produtos de higiene e limpeza. Essa última ação teria como objetivo manter localizado um foco de contaminação, sem comprometer a atividade dos demais bombeiros (Montrose, 2020).

Diante do exposto até aqui, podemos enumerar alguns pontos importantes a serem considerados quando se trata dos desafios e dificuldades enfrentadas pelos profissionais do Corpo de Bombeiros. Nota-se que, diante dessa crise sanitária sem precedentes, a falta de trabalhadores no suporte à saúde foi um entrave no combate à pandemia enfrentado por muitos países. No Brasil, a integração do Corpo de Bombeiros se mostrou adequada e imprescindível, uma vez que esses profissionais são treinados e capacitados para salvar vidas. No entanto, lidar com um desastre biológico de natureza infecciosa e letal não é uma atividade corriqueira para o Corpo de Bombeiros. Nesse sentido, o Samu e as Secretarias de Saúde de alguns Estados brasileiros, como Bahia (CBM-BA, 2020) e Santa Catarina (Malinverni, 2020) já estão dando suporte a esses profissionais por meio de treinamentos para o uso de equipamentos de proteção individual, apoio psicológico e monitoramento das taxas de infecção ao longo do tempo.

\section{Discussão}

As ações desempenhadas pelos bombeiros variaram de acordo com as demandas estaduais e municipais, seja no transporte de pacientes, nas ações de sanitização do município ou na testagem em massa da população. Cada uma dessas atividades representa riscos maiores ou menores de exposição ao vírus e exigem protocolos de segurança específicos a serem seguidos. É evidente que uma das principais dificuldades dos bombeiros no combate à pandemia esteve relacionada com sua própria proteção, visto o número de servidores infectados e afastados de suas atividades, além dos que vieram a óbito. Assim como médicos e enfermeiros, os bombeiros enfrentam diariamente o risco de contaminação, mesmo com o uso de equipamentos de proteção individual. Isso porque acontecem falhas ao seguir com rigor os protocolos de segurança por diversos motivos, entre eles: a sobrecarga de trabalho e o estresse em situação de risco, bem como a falta de equipamentos de proteção que deveriam ser fornecidos em quantidade e qualidade adequadas (Huh, 2020). Diante disso, cursos de capacitação são suficientes para promover a saúde e qualidade de vida destes trabalhadores.

\section{Considerações finais}

De acordo com as diversas fontes utilizadas neste estudo, pode-se verificar que conviver com a Covid-19 é adaptar-se a um novo estilo de vida para prevenir a reincidência da doença até o surgimento da vacina para a população. Portanto, os efeitos da pandemia deverão ser sentidos por muito tempo no futuro, principalmente com relação à saúde mental de todos os profissionais da saúde. No caso dos bombeiros, medidas de suporte de apoio emocional com psicólogos e outras formas de assistência médica e social aos profissionais são fundamentais e já têm sido realizadas em alguns Estados como Bahia e Santa Catarina. Essas medidas precisam ser ampliadas e continuadas, mesmo após a superação da pandemia.

\section{Referências}

Almeida, I. M. 2020. Proteção da saúde dos trabalhadores da saúde em tempos de COVID-19 e respostas à pandemia. Revista Brasileira de Saúde Ocupacional, 45, 1-10.

Andrade, V. 2020. RJ: Corpo de Bombeiros afasta 628 agentes por covid-19. Rio de Janeiro, RJ: R7. Disponível em: https://noticias.r7.com/saude/coronavirus/rj-corpo-de-bombeiros-afasta-628-agentes-por-covid19-11052020. Acesso em: 16 set. 2020.

Baker, M. G., Peckham, T. K. \& Seixas, N. S. 2020. Estimating the burden of United States workers exposed to infection or disease: A key factor in containing risk of COVID-19 infection. PLOS ONE, 15, 1-8.

CBM-BA. 2020. Ações de suporte aos bombeiros militares durante a crise do novo coronavírus. Disponível em: http://www.cbm.ba.gov.br/noticias/acoes-de-suporte-aos-bombeiros-militares-durante-crise-do-novocoronavirus. Acesso em: 16 set. 2020. 
CBM-PB. 2020. Corpo de bombeiros estrutura ações de prevenção e atendimento em casos de covid-19. Disponível em: https://bombeiros.pb.gov.br/corpo-de-bombeiros-estrutura-acoes-de-prevencao-eatendimento-em-casos-de-covid-19/?utm_source=rss \&utm_medium=rss \&utm_campaign=corpo-debombeiros-estrutura-acoes-de-prevencao-e-atendimento-em-casos-de-covid-19. Acesso em: 16 set. 2020.

CCB-BM1. 2020. Dados do Departamento Pessoal do Corpo de Bombeiros do Paraná no Combate à COVID-19. Disponível em: http://boletinsccb.bombeiros.parana/BM1/COVID19/. Acesso em: 25 set. 2020.

Cespedes, M. S. \& Souza, J.C.R.P. 2020. Sars-CoV-2: A clinical update - II. Revista Associação Médica Brasileira, 66, 547-557.

Correia, G., Rodrigues, L., Silva, M. G., \& Gonçalves, T. 2020. Airborne route and bad use of ventilation systems as non-negligible factors in SARS-CoV-2 transmission. Medical Hypotheses, 141, 109781-109785.

Elias, F. 2020. Avanço da covid-19 ocasiona fechamento de pelotão do corpo de bombeiros em Blumenau. Disponível em: https://ocp.news/seguranca/avanco-da-covid-19-ocasiona-fechamento-de-pelotao-docorpo-de-bombeiros-em-blumenau. Acesso em: 16 set. 2020.

Em Dia. 2020. Em parceria com o Detran/ES, Corpo de Bombeiros adquire 20 viaturas para resgatar vítimas de acidentes. Disponível em: http://www.linharesemdia.com.br/Noticias/Utilidades/em-parceria-com-odetranes-corpo-de-bombeiros-adquire-20-viaturas-para-resgatar-vitimas-de-acidentes. Acesso em: 16 set. 2020.

Ferraz, R. 2020. Samu se une ao corpo de bombeiros para reforçar atendimento. Disponível em: https:/www.agenciabrasilia.df.gov.br/2020/07/11/samu-se-une-ao-corpo-de-bombeiros-para-reforcaratendimento/. Acesso em: 16 set. 2020.

Freitas, A. R. R., Napimoga, M. \& Donalisio, M. R. 2020. Análise da gravidade da pandemia de Covid-19. Epidemiologia e Serviços da Saúde, 29, 1-5.

Frias, S. 2020. Drive-thru do Corpo de Bombeiros passa a fazer testagem da covid-19 à noite. Disponível em: https://www.campograndenews.com.br/cidades/capital/drive-thru-do-corpo-de-bombeiros-passa-afazer-testagem-da-covid-19-a-noite. Acesso em: 16 set. 2020.

G1-DF. 2020. Corpo de Bombeiros do DF tem 9 militares com coronavírus; 4 são de Águas Claras. Disponível em: https://g1.globo.com/df/distrito-federal/noticia/2020/04/21/corpo-de-bombeiros-do-df-tem-9militares-com-coronavirus-4-sao-de-aguas-claras.ghtml. Acesso em: 16 set. 2020.

Gondim, R. 2020. Bombeiros fazem teste de Covid-19. Disponível em: http://portal.rr.gov.br/index.php/component/k2/item/2276-bombeiros-fazem-teste-de-covid-19. Acesso em: 16 set. 2020.

Greenberg, N., Docherty, M., Gnanapragasam, S., Wessel, Y. S. 2020. Managing mental health challenges faced by healthcare workers during covid-19 pandemic. The BMJ, 368, 1-4.

Huh, S. 2020. How to train health personnel to protect themselves from SARS-CoV-2 (novel coronavirus) infection when caring for a patient or suspected case. Journal of Educational Evaluation for Health Professions, 17, 1-10.

Liu, M., He, P., Li, H. G., Wang, X. J., Li, F. J., Chen, P., Liu, J. H., Li, C. H. 2020. Clinical characteristics of 30 medical workers infected with new coronavirus pneumonia. Chinese Journal of Tuberculosis and Respiratory Diseases, 43, 209-214.

Machado, L. H. 2020. Na linha de frente contra a covid-19, bombeiros militares não retrocedem. Disponível em: https://bombeiros.to.gov.br/noticia/2020/7/2/na-linha-de-frente-contra-a-covid-19-bombeirosmilitares-nao-retrocedem/. Acesso em: 16 set. 2020.

Malinverni, R. N. 2020. DSPS oferece atendimento exclusivo aos policiais durante a quarentena. Polícia Militar de Santa Catarina, Florianópolis. Disponível em: https://www.pm.sc.gov.br/noticias/dsps-ofereceatendimento-exclusivo-aos-policiais-durante-quarentena. Acesso em: 16 set. 2020.

Moghadas, S. M., Shoukat, A., Fitzpatrick, M. C., Wells, C. R., Sah, P., Pandey, A., Sachs, J. D., Wang, Z., Meyers, L. A., Singer, B. H., Galvani, A. P. 2020. Projecting hospital utilization during the COVID-19 outbreaks in the United States. Proceedings of the National Academy of Sciences of the USA, 117, 9122-6.

Montrose, L. 2020. Smoke from wildfires can worsen COVID-19 risk, putting firefighters in even more danger. Disponível em: https://theconversation.com/smoke-from-wildfires-can-worsen-covid-19-risk-puttingfirefighters-in-even-more-danger-145998?fbclid=IwAR04N1DPa_MPCRViTJa6DDQELFeRVKzeCey5WuI7F_WLyBo5ms3UwsUPoY. Acesso em: 16 set. 2020. 
ND mais. 2020. Florianópolis fará testes da Covid-10 em militares e bombeiros. Disponível em: https://ndmais.com.br/saude/florianopolis-fara-testes-da-covid-19-em-militares-e-bombeiros/. Acesso em: 16 set. 2020.

Oliveira, C. 2020. Unidades de resgate dos bombeiros poderão ser usadas para transportar pacientes com Covid em Minas. Disponível em: https://www.hojeemdia.com.br/horizontes/unidades-de-resgate-dosbombeiros-poder\%C3\%A3o-ser-usadas-para-transportar-pacientes-com-covid-em-minas-1.792797. Acesso em: 16 set. 2020.

Pimentel, I. 2020. Covid: 14 bombeiros no Piauí estão em isolamento após testarem positivo. Cidade Verde. Disponível em: https://cidadeverde.com/coronavirus/108718/covid-14-bombeiros-no-piaui-estao-emisolamento-apos-testarem-positivo. Acesso em: 16 set. 2020.

Poços Já. 2020. Mais de cem bombeiros de Poços são testados para covid-19. Disponível em: https://www.pocosja.com.br/2020/08/17/mais-de-cem-bombeiros-de-pocos-sao-testados-para-covid19/. Acesso em: 16 set. 2020.

Relatório Mundial De Saúde. 2006. Manual para a monitorização de recursos humanos de saúde: com aplicação dedicada aos países de rendimento baixo e médio. Genebra, SW: OMS.

Sant'ana G., Imoto, A. M., Amorim, F. F., Taminato, M., Peccin, M. S., Santana, L. A., Göttems, L. B. D., Camargo, E. B. 2020. Infecção e óbitos de profissionais da saúde por COVID-19: revisão sistemática. Acta Paulista de Enfermagem, 33, 1-9.

Shereen, M. A., Khan, S., Kazmi, A., Bashir, N., Siddique, R. 2020. COVID-19 infection: origin, transmission, and characteristics of human coronaviruses. Journal of Advanced Research, 24, 91-8.

Silva, J. M. \& Nobre, L. C. C. 2020. A Covid-19, a saúde e bem-estar dos trabalhadores: a barbárie entre nós. Disponível em: https://www.abrasco.org.br/site/noticias/sistemas-de-saude/a-covid-19-a-saude-e-bemestar-dos-trabalhadores-a-barbarie-entre-nos/47465/. Acesso em: 18 set. 2020.

Troyer, E. A., Kohn, J. N. \& Hong, S. 2020. Are we facing a crashing wave of neuropsychiatric sequelae of COVID-19? Neuropsychiatric symptoms and potential immunologic mechanisms. Brain, Behavior, and Immunity, 87, 34-9.

World Health Organization [WHO]. 2020. Coronavirus disease 2019 (COVID-19): Situation report. Disponível em: https:/www.who.int/docs/default-source/coronaviruse/situation-reports/20200311-sitrep-51-covid19.pdf?sfvrsn=1ba62e57_10. Acesso em: 16 set. 2020.

\section{Minicurrículo}

Vinícius Sonoda de Freitas. Graduação em Educação Física pelo Centro Universitário de Maringá Unicesumar. Especialista em Saúde e Atividade Física pelo Centro Universitário de Maringá - Unicesumar. Servidor Público pelo Corpo de Bombeiros do Paraná.

Como citar: Freitas, V.S. 2020. A saúde dos bombeiros militares no combate a COVID19 no Brasil. Pubsaúde, 4, a083. DOI: https://dx.doi.org/10.31533/pubsaude4.a083

Recebido: 2 out. 2020.

Revisado e aceito: 6 out. 2020 .

Conflito de interesse: os autores declaram, em relação aos produtos e companhias descritos nesse artigo, não ter interesses associativos, comerciais, de propriedade ou financeiros que representem conflito de interesse.

Licenciamento: Este artigo é publicado na modalidade Acesso Aberto sob a licença Creative Commons Atribuição 4.0 (CC-BY 4.0). 